\title{
A review of the literature: direct and video laryngoscopy with simulation as educational intervention
}

This article was published in the following Dove Press journal:

Advances in Medical Education and Practice

28 January 2014

Number of times this article has been viewed

\section{Allison A Vanderbilt Julie Mayglothling' \\ Nicholas J Pastis ${ }^{2}$ \\ Douglas Franzen ${ }^{3}$}

'School of Medicine, Virginia Commonwealth University, Richmond, VA, ${ }^{2}$ Division of Pulmonary and Critical Care, Medical University of South Carolina, SC, ${ }^{3}$ School of Medicine, University of Washington, WA, USA
Correspondence: Allison A Vanderbilt School of Medicine, Virginia

Commonwealth University, 730 East Broad St Suite 4200 Box 980466 .

Richmond, VA 23298, USA

Tel +l 8048278490

$\mathrm{Fax}+\mathrm{I} 8048270663$

Email avanderbilt@vcu.edu
Introduction: A review of the literature was conducted to analyze the impact of simulation-based training for direct and video laryngoscopy (VL) skills for health care professionals and health care students.

Methods: This review focused on the published literature that used randomized controlled trials to examine the effectiveness of simulation-based training to develop airway management skills and identify pertinent literature by searching PubMed from inception of the database up to July 2013. This current review addresses the question of whether airway management simulation-based training improves the acquisition of resuscitation skills for health care profession learners.

Results: A total of eleven articles qualified for this systematic review based on the inclusion and exclusion criteria. These studies were analyzed and the specific simulators, participants, assessments, and details related to: time of intubation; Cormack and Lehane classification; success and failure rate; and number of attempts.

Conclusion: This review suggests that simulation-based training is one effective way to teach VL skills. VL allows for a higher success rate, faster response time, and a decrease in the number of attempts by health care students and health care professionals under the conditions based on the eleven studies reviewed.

Keywords: laryngoscopy, video laryngoscopy, simulation, systematic review, health care professionals, health care students

\section{Introduction}

More than 400,000 Americans die annually from sudden cardiac arrest. ${ }^{1}$ Sudden cardiac death is a serious medical problem ${ }^{2}$ and it is critical that there is a rapid response because it influences survival outcomes for the patient; each additional minute of delayed defibrillation will reduce survival in cardiac arrest by $7 \%-10 \%$. Airway management is a fundamental skill set for health care professionals. It is reported that annually in Canada 100 to 700 real life events for airway management lead to cannot intubate or cannot ventilate situations. ${ }^{3}$ Scientific evidence for the treatment of cardiac arrest focuses on medical expertise, chest compressions, early defibrillation, and hyperventilation avoidance. ${ }^{4,5}$

Unfortunately, a significant proportion of deaths from injury are considered preventable $^{6}$ due to the lack of airway management support. According to Batchelder et al, many of these injuries are from failure to identify and treat life-threatening injuries promptly in the pre-hospital phase of care. ${ }^{7}$ The National Confidential Enquiry into Patient Outcomes and Death concluded that the current structure of pre-hospital management is insufficient to meet the needs of the severely injured patient and that 
the pre-hospital response should include someone with the skill to rescue the airway and maintain adequate ventilation. ${ }^{8}$ Inadequate airway management for endotracheal intubation in a pre-hospital setting is the primary cause of preventable mortality. ${ }^{9}$ There continues to be adverse outcomes related to mismanagement of cannot intubate and cannot ventilate situations, such as death and brain damage. ${ }^{10-12}$

Simulation-based education has been implemented in many training sub specialties such as anesthesia, emergency medicine, and surgery ${ }^{13}$ and with medical students. ${ }^{14}$ Although simulations have been effective in training programs, the rigor and quality of research in the field still needs improvement. ${ }^{13}$ Simulation can assist with the analysis of medical knowledge and other factors that influence the delivery of adequate care in cardiopulmonary arrest. ${ }^{15}$

Direct laryngoscopy (DL) is a difficult skill to master ${ }^{16}$ and requires multiple exposures and attempts to acquire the skills necessary to replicate DL successfully on a patient. Narang et $\mathrm{al}^{17}$ argue the unanticipated difficult airway can be risky for the patient; thus requiring great training on behalf of the physician. However, video laryngoscopy (VL) has shown improvement with glottis exposure compared to $\mathrm{DL}^{18}$ and VL has been developed to manage difficult airways. ${ }^{19}$ The purpose of this manuscript is to review the literature and compare DL with VL using simulation as an education intervention.

\section{Methods}

We focused on the published literature that examined the effectiveness of DL and VL with simulation-based training as the educational intervention. Studies were identified by searching PubMed, from the inception of the database to July 2013. Multiple combinations of several relevant medical subject headings (MeSH) terms were used to identify articles for review (laryngoscopy, laryngoscopy and simulation; video laryngoscopy, laryngoscopy and simulation). This resulted in a total of 1,152 published manuscripts to review. The inclusion and exclusion criteria address how these manuscripts were eliminated for our search to eleven.

\section{Inclusion and exclusion criteria}

Inclusion criteria required that studies: a) use a randomized controlled design; b) single-group pretest-posttest; c) two group nonrandomized; d) parallel group; e) crossover designs; and $\mathrm{f}$ ) used simulation-based training as the educational intervention. Simulation-based training was defined broadly to range from task trainers to high fidelity simulators. The exclusion criteria were: a) opinion or commentary literature; b) not a study; and c) studies that did not use simulation as the educational intervention.

\section{Data extraction and analysis}

Two authors read the literature and independently determined if the article should be included in the review based on the previously stated inclusion criteria. When reviewing the literature, some abstracts provided enough detail and information related to the methods to determine if the inclusion criteria were met; if not, the full manuscript was then read to determine if the methods met the inclusion criteria. The manuscripts in which the methods did not meet the inclusion criteria were excluded. The two reviewers met to discuss the included and excluded manuscripts. All differences with respect to inclusion of a study were resolved with unanimity as the final criterion.

\section{Results}

A total of eleven articles were identified for this review based on the inclusion and exclusion criteria to compare DL and VL. These studies were analyzed and the specific simulators and/or task trainers, participants, assessments, and details of the eleven studies are provided in both Tables 1 and 2 .

The average amount of time the participants received on training with the VL was 7.6 minutes (ranging from 1-12 minutes, standard deviation $[\mathrm{SD}]=4.51$ ). In two studies the participants did not practice $(n=2),{ }^{20,22}$ whereas, two studies allowed the participants to practice a simulated VL until they felt competent. ${ }^{24,25}$ Following the training of the participants with simulation and VL they were given an assessment. These assessments include the following: two studies in the emergency department (18\%), one study with the patient on the floor $(9 \%)$, one study in the ambulance stations and emergency departments (9\%), one study in a simulation lab (9\%), one study at a major trauma scene $(9 \%)$, and five studies whose location was not specified (45\%). Finally, three studies (27\%) used automated data collected from the simulator for their outcome data. The majority of the studies used observational ratings (82\%) and participant self-report (55\%) as the source for assessment.

\section{Time of intubation}

Time of intubation $(n=8)$ was reported as the amount of time to perform a successful intubation. Of the eight studies (38\%) that assessed whether time of intubation increased when using VL, three reported statistically significant improvement in time to intubate. For instance, one study described the median time to intubation as 25 seconds 


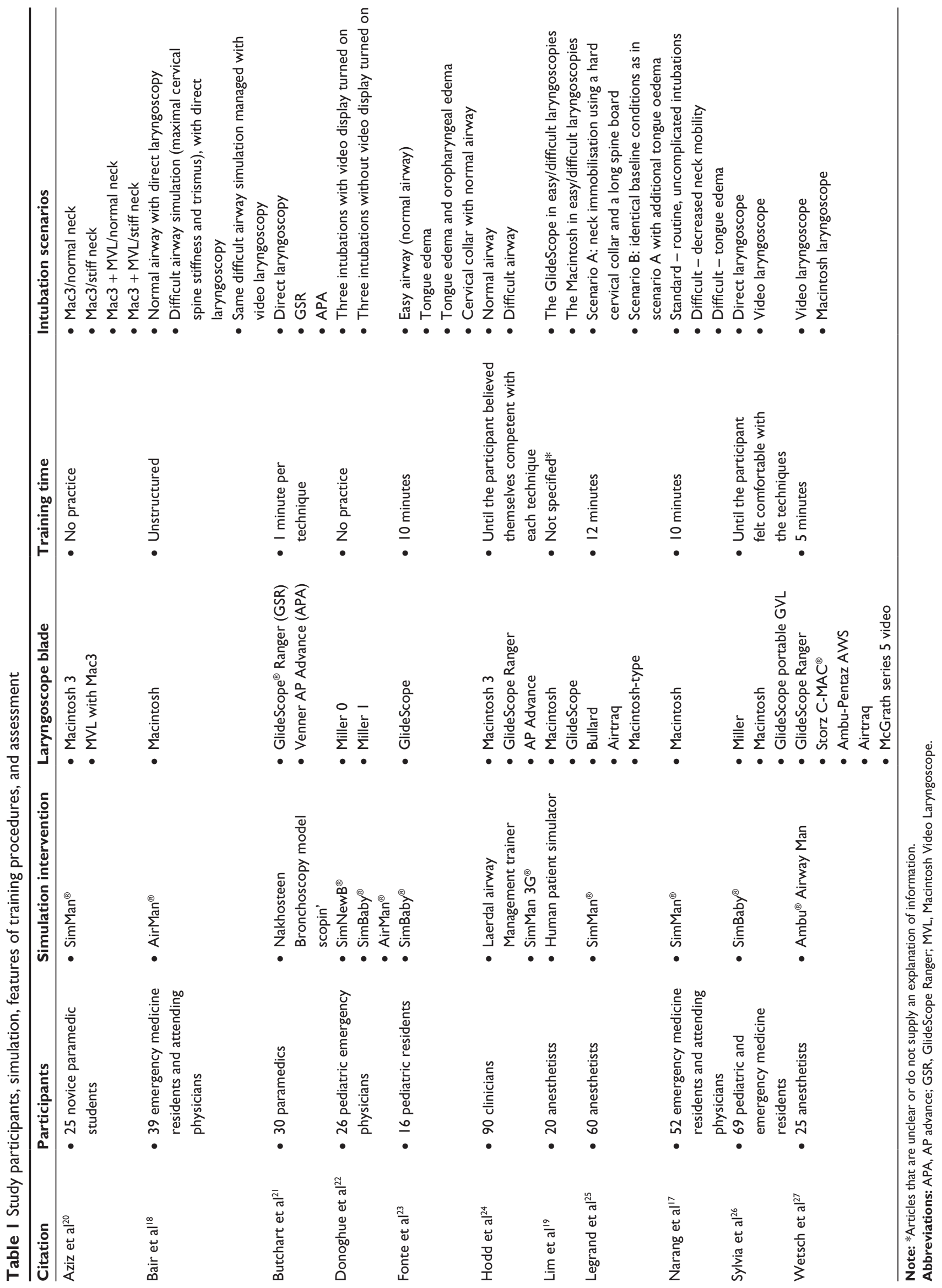




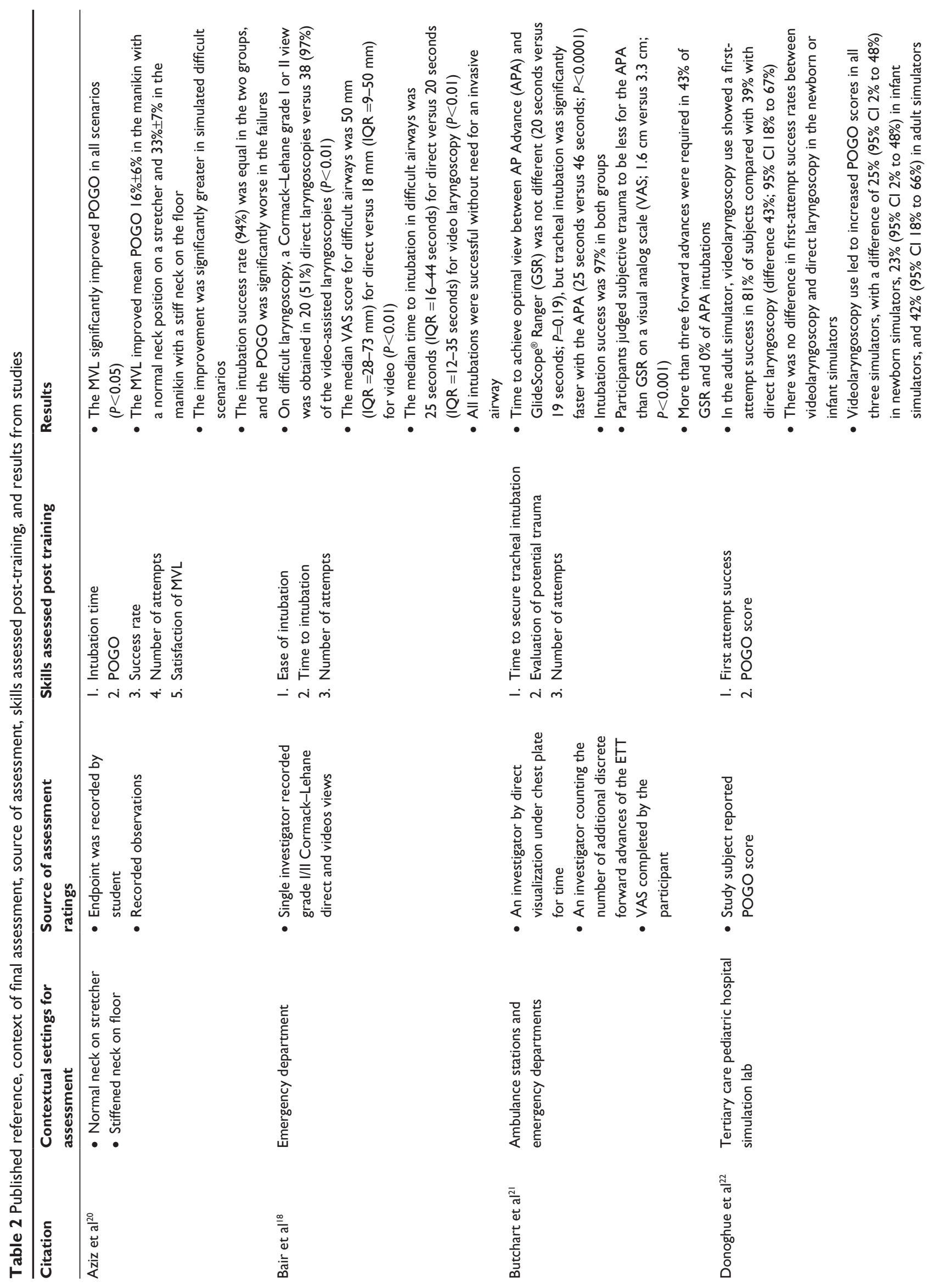



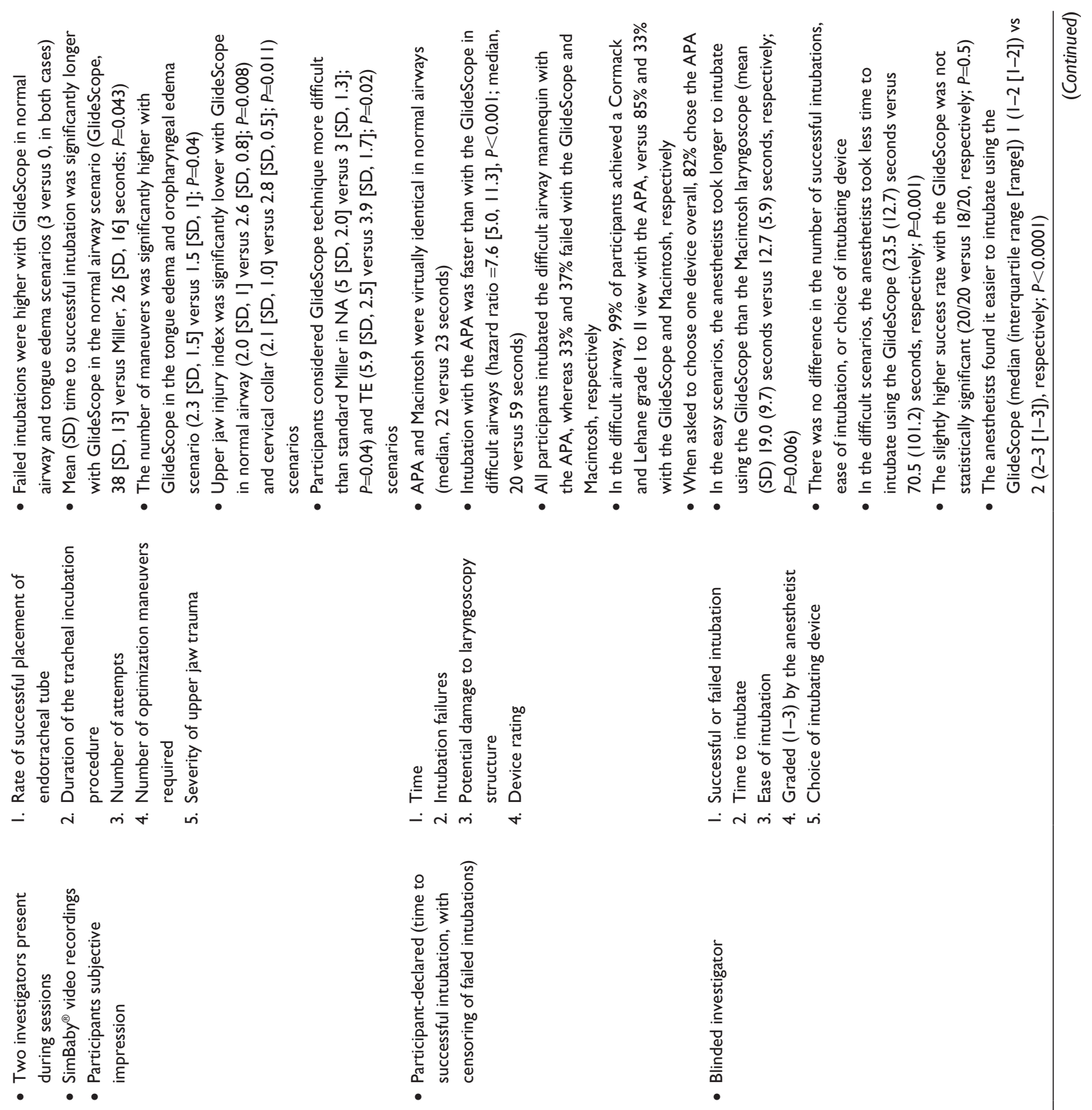

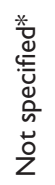

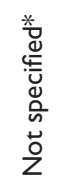

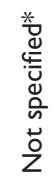

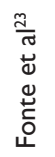

I
$\frac{0}{0}$
$\frac{0}{0}$
$\frac{0}{0}$
$\frac{0}{4}$

$\frac{a}{\bar{J}}$
$\stackrel{0}{0}$
$\stackrel{\Xi}{\Xi}$ 


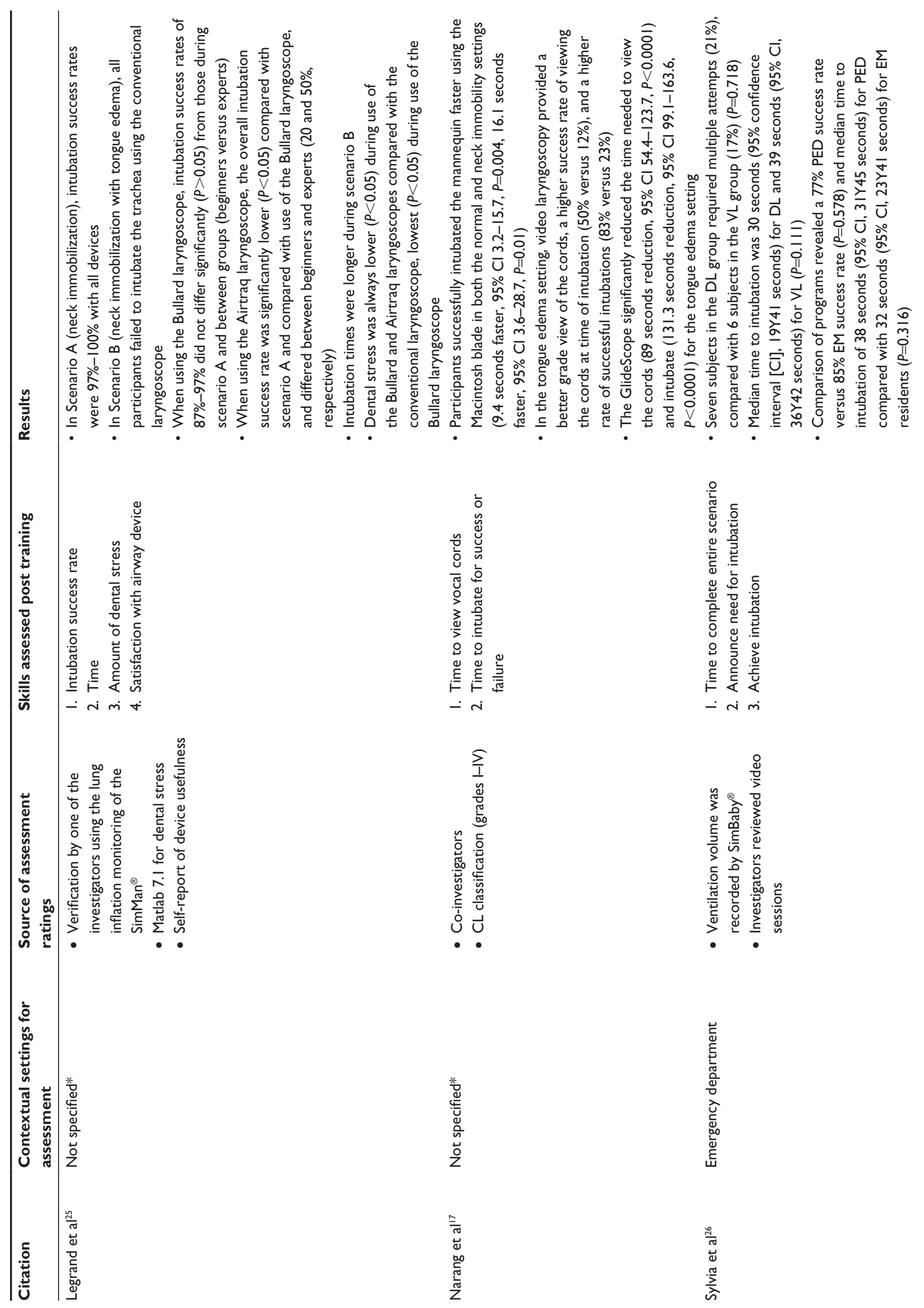



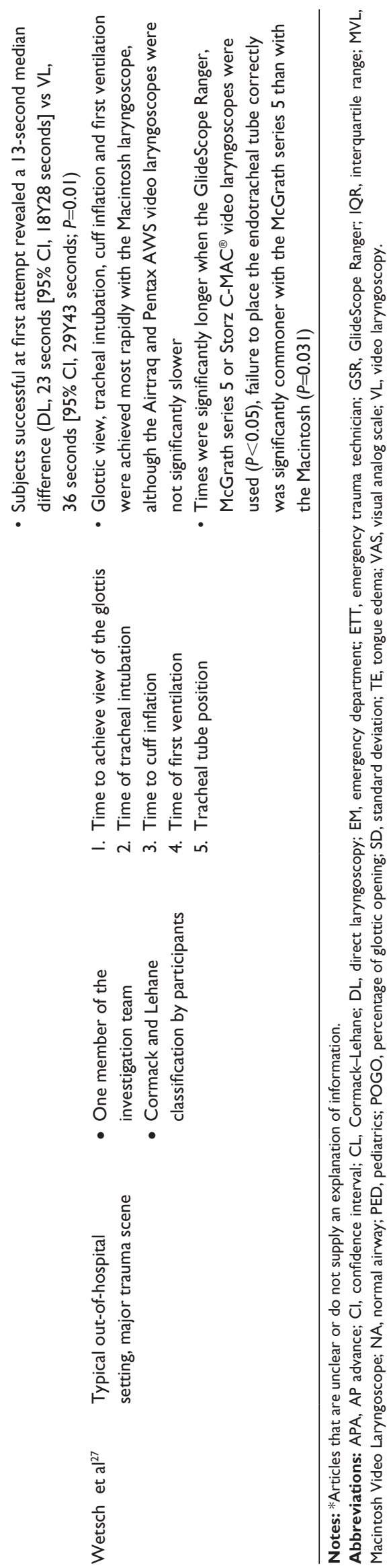

(interquartile range $[\mathrm{IQR}]=16-44$ seconds) using $\mathrm{DL}$ and 20 seconds (IQR $=12-35$ seconds) for $\mathrm{VL}(P<0.01) .{ }^{18} \mathrm{In}$ another study, the anesthetists took less time to intubate, in the difficult scenarios, when using the GlideScope than the Macintosh laryngoscope 23.5 (12.7) seconds versus 70.5 (101.2) seconds, respectively $(P=0.001) .{ }^{19}$ Another study reported that intubation with the AP Advance was faster than with the GlideScope in difficult airways (median, 20 versus 59 seconds, $P<0.001) .{ }^{24}$ On the other hand, four studies reported VL taking longer to intubate than DL. 17,23,26,27 One study, did not explicitly report their significant or nonsignificant findings. ${ }^{20}$

\section{Cormack and Lehane classification}

The Cormack-Lehane ( $\mathrm{CL} ; \mathrm{n}=4)$ grading system is used to assess and quantify the laryngoscopic view of the vocal cords and glottis. The complete exposure of the glottis, or the best view, is defined as CL grade I whereas, in CL grade IV, neither the glottis nor epiglottis can be seen. In three of the four studies $(75 \%)$, researchers reported that the VL provided a better grade view. For example, one study found that during a difficult laryngoscopy, a CL grade of I or II view was obtained in only 20 (51\%) DL versus 38 (97\%) of the video-assisted laryngoscopies $(P<0.01) .{ }^{18}$ In addition, the VL provided a better grade view of the cords and significantly reduced the time needed to view the cords ( 89 second reduction; $P<0.0001)$ in another study. ${ }^{17}$ Similarly, another study found that in the difficult airway scenario, $99 \%$ of the participants achieved a CL grade I to II view with the AP Advance VL, versus $85 \%$ and $33 \%$ with the GlideScope and Macintosh, respectively. ${ }^{24}$ In the last study, the glottic view was achieved most rapidly with the Macintosh laryngoscope, although it was not significantly faster than the VL. ${ }^{27}$

\section{Success and failure rate}

Seven of the eleven studies evaluated the rate of successful or failed intubations. Automated data or an investigator assessed a successful intubation as the tube being passed through the vocal cords in the mannequin. Thus, a failed intubation is the tube not passing through the vocal cords to allow an open airway. One of the seven studies (14\%) reported a statistically significant increase in success rate while using the VL. During the difficult scenario in this study, all participants failed to intubate the trachea using the conventional laryngoscope. The same study examined two different VL devices in addition to the conventional method. While using the Bullard laryngoscope, the success rate was significantly higher $(P<0.05)$ than when the participants used the Airtraq laryngoscope. ${ }^{25}$ 
Two of the seven studies (29\%) found an increase in failure rates and a decrease in success rates while using the VL. Fonte et al reported higher failed intubations with the GlideScope in normal airway and tongue edema scenarios ( 3 versus 0 , in both cases). ${ }^{23}$ In another study, there was a 13 -second median difference during the first successful attempt (DL, 23 seconds versus VL 36 seconds, $P=0.01) .{ }^{26}$ The majority of the studies (57\%) did not find significant differences in intubation success rates in DL and VL procedures. ${ }^{18-21}$

\section{Number of attempts}

Three of the eleven total studies $(27 \%)$ reported on the number of attempts to successfully intubate. All three studies $(100 \%)$ found a decrease in the number of attempts to intubate while using a VL. More specifically, the VL used during the adult simulation showed a first-attempt success in $81 \%$ of subjects compared with $39 \%$ with DL (a difference of $43 \%){ }^{22}$ In a different study, more than three forward advances were required in $43 \%$ of GlideScope Ranger and $0 \%$ of the AP Advance VL intubations. ${ }^{21}$ Although not statistically significant, Sylvia et al had seven subjects in the DL group require multiple attempts $(21 \%)$ compared with the six subjects in the VL group $(17 \% ; P=0.718) .{ }^{26}$

\section{Discussion}

This review of laryngoscopy research adds evidence that simulation-based training can result in skill transfer, providing a safe and effective way for health care professionals to practice and for health care students to learn. Additionally, it is evident based on the review of the literature that students learn faster, have fewer errors, and require fewer attempts when using a VL. VL has the potential to replace the traditional DL as an educational tool with simulation; however, additional research is needed. Potentially in the future, VL may replace DL in all settings, but the authors would caution against not teaching DL. DL is still an important skill to know and have should there be a potential natural disaster or crisis when DL is the only skill available.

Future research needs to focus on a larger sample size, maintenance of skills, and patient outcomes. In order to determine that VL may be a better method of accessing the throat, vocal cords, and airway it is essential that data be collected on patient outcomes.

\section{Limitations}

As with any systematic review, our review and results are limited by the data provided in the original studies. Thus, despite the adequate number of relevant studies, the studies included in this systematic review provide only a limited basis for examining the impact of simulation in laryngoscopy skills training. Our findings are also limited by the lack of descriptions of the data collection process and interventions of the included studies. The lack of effect size reporting contributes to the difficulty in truly understanding the magnitude of the effect of these interventions on the acquisition of laryngoscopy skills.

The scope of our review is both a strength and limitation. However, it is not possible to draw firm conclusions about the effectiveness of the different types of simulation based on this review. Nonetheless, we argue that our review does provide useful insight into the literature that examines the effectiveness of simulation-based laryngoscopy training interventions. The need for more robust examinations of these training interventions is needed to be able to provide an unequivocal conclusion to the impact on learning, maintenance of skills, and potentially better outcomes for patients.

\section{Conclusion}

This review suggests that simulation-based training is one effective way to teach VL laryngoscopy skills. VL allows for a higher success rate, faster response time, and a decrease in the number of attempts by health care students and health care professionals under the conditions based on the eleven studies reviewed. The findings from this initial review of the literature VL have the potential to be a more effective way to view a patient's throat, vocal cords, and airway.

\section{Disclosure}

The authors report no conflicts of interest in this work.

\section{References}

1. Rubart M, Zipes DP. Mechanisms of sudden cardiac death. J Clin Invest. 2005;115(9):2305-2315.

2. Demaria S, Bryson EO, Mooney TJ, et al. Adding emotional stressors to training in simulated cardiopulmonary arrest enhances participant performance. Med Educ. 2010;44(10):1006-1015.

3. Crosby E. The unanticipated difficult airway - evolving strategies for successful salvage. Can J Anaesth. 2005;52(6):562-567.

4. Aufderheide TP, Lurie KG. Death by hyperventilation: a common and life-threatening problem during cardiopulmonary resuscitation. Crit Care Med. 2004;32(Suppl 9):S345-S351.

5. Eftestøl T, Wik L, Sunde K, Steen PA. Effects of cardiopulmonary resuscitation on predictors of ventricular fibrillation defibrillation success during out-of-hospital cardiac arrest. Circulation. 2004;110(1): 10-15.

6. Royal College of Surgeons of England and British Orthopaedic Association. Better Care for the Severely Injured. London: Royal College of Surgeons of England; 2000.

7. Batchelder AJ, Steel A, Mackenzie R, Hormis AP, Daniels TS, Holding N. Simulation as a tool to improve the safety of pre-hospital anaesthesia - a pilot study. Anaesthesia. 2009;64(9):978-983.

8. Henning DC, Smith JE, Patch D, Lambert AW. A comparison of civilian (National Confidential Enquiry into Patient Outcome and Death) trauma standards with current practice in a deployed field hospital in Afghanistan. Emerg Med J. 2011;28(4):310-312. 
9. Adnet F, Lapostolle F, Ricard-Hibon A, Carli P, Goldstein P. Intubating trauma patients before reaching hospital - revisited. Crit Care. 2001;5(6):290-291.

10. Cheney FW, Posner KL, Lee LA, Caplan RA, Domino KB. Trends in anesthesia-related death and brain damage: A closed claims analysis. Anesthesiology. 2006;105(6):1081-1086.

11. Peterson GN, Domino KB, Caplan RA, Posner KL, Lee LA, Cheney FW. Management of the difficult airway: a closed claims analysis. Anesthesiology. 2005;103(1):33-39.

12. Berkow LC, Greenberg RS, Kan KH, et al. Need for emergency surgical airway reduced by a comprehensive difficult airway program. Anesth Analg. 2009;109(6):1860-1869.

13. Issenberg SB, McGaghie WC, Petrusa ER, Lee Gordon D, Scalese RJ. Features and uses of high-fidelity medical simulations that lead to effective learning: a BEME systematic review. Med Teach. 2005;27(1):10-28.

14. Pastis NJ, Doelken P, Vanderbilt AA, Walker J, Schaefer JJ. Validation of simulated difficult bag-mask ventilation as a training and evaluation method for first-year internal medicine house staff. Simul Healthc. 2013;8(1):20-24.

15. Gaba DM, DeAnda A. A comprehensive anesthesia simulation environment: re-creating the operating room for research and training. Anesthesiology. 1988;69(3):387-394.

16. Mulcaster JT, Mills J, Hung OR, et al. Laryngoscopic intubation learning and performance. Anesthesiology. 2003;98(1):23-27.

17. Narang AT, Oldeg PF, Medzon R, MahmoodAR, Spector JA, Robinett DA. Comparison of intubation success of video laryngoscopy versus direct laryngoscopy in the difficult airway using high-fidelity simulation. Simul Healthc. 2009;4(3):160-165.

18. Bair AE, Olmsted K, Brown CA, Barker T, Pallin D, Walls RM. Assessment of the storz video Macintosh laryngoscope for use in difficult airways: A human simulator study. Acad Emerg Med. 2010;17(10): 1134-1137.

19. Lim TJ, Lim Y, Liu EH. Evaluation of ease of intubation with the GlideScope or Macintosh laryngoscope by anaesthetists in simulated easy and difficult laryngoscopy. Anaesthesia. 2005;60(2): 180-183.
20. Aziz M, Dillman D, Kirsch JR, Brambrink A. Video laryngoscopy with the macintosh video laryngoscope in simulated prehospital scenarios by paramedic students. Prehosp Emerg Care. 2009;13(2): 251-255.

21. Butchart AG, Tjen C, Garg A, Young P. Paramedic laryngoscopy in the simulated difficult airway: comparison of the Venner AP Advance and GlideScope Ranger video laryngoscopes. Acad Emerg Med. 2011;18(7):692-698.

22. Donoghue AJ, Ades AM, Nishisaki A, Deutsch ES. Videolaryngoscopy versus direct laryngoscopy in simulated pediatric intubation. Ann Emerg Med. 2013;61(3):271-277.

23. Fonte M, Oulego-Erroz I, Nadkarni L, Sánchez-Santos L, Iglesias-Vásquez A, Rodríguez-Núñez A. A randomized comparison of the GlideScope videolaryngoscope to the standard laryngoscopy for intubation by pediatric residents in simulated easy and difficult infant airway scenarios. Pediatr Emerg Care. 2011;27(5):398-402.

24. Hodd JA, Doyle DJ, Gupta S, et al. A mannequin study of intubation with the AP advance and GlideScope Ranger videolaryngoscopes and the Macintosh laryngoscope. Anesth Analg. 2011;113(4):791-800.

25. Legrand MA, Steinmann D, Priebe HJ, Mols G. Comparison of Bullard and Airtraq laryngoscopes with conventional laryngoscopy in a manikin study of simulated difficult intubation. Eur J Anaesthesiol. 2012;29(7):343-350.

26. Sylvia MJ, Maranda L, Harris KL, Thompson J, Walsh BM. Comparison of success rates using video laryngoscopy versus direct laryngoscopy by residents during a simulated pediatric emergency. Simul Healthc. 2013;8(3):155-161.

27. Wetsch WA, Carlitscheck M, Spelten O, et al. Success rates and endotracheal tube insertion times of experienced emergency physicians using five video laryngoscopes: a randomised trial in a simulated trapped car accident victim. Eur J Anaesthesiol. 2011;28(12):849-858.
Advances in Medical Education and Practice

\section{Publish your work in this journal}

Advances in Medical Education and Practice is an international, peerreviewed, open access journal that aims to present and publish research on Medical Education covering medical, dental, nursing and allied health care professional education. The journal covers undergraduate education, postgraduate training and continuing medical education

\section{Dovepress}

including emerging trends and innovative models linking education, research, and health care services. The manuscript management system is completely online and includes a very quick and fair peer-review system. Visit http://www.dovepress.com/testimonials.php to read real quotes from published authors. 